O26 (continued)

could be grown in the deep fertile soil of the Mississippi Delta.

Funding: USDA, USDA ARS.

\section{Appreciation for Food Mediates the Association of Meal-Related Rituals Learned in Childhood with Avoiding Food Waste Behavior in Japanese Adults}

\author{
Yui Kawasaki, PhD, Registered Dietitian (Japan), \\ kawasaki@uni-potsdam.de, University of Potsdam, Karl- \\ Liebknechtstr 24-25, Potsdam, Brandenburg, Germany, \\ 14476; Rie Akamatsu, DrPH, Registered Dietitian (Japan), \\ Ochanomizu University; Petra Warschburger, PhD, \\ University of Potsdam
}

Background: Saying the words "Itadakimasu" and "Gochisosama" is a common Japanese ritual at the beginning and end of a meal. Many Japanese parents and educators communicate to children to express appreciation for food on a daily basis through these words, and they habitually connect these rituals to further education to avoid food waste to children.

Objective: This study aimed to examine whether appreciation for food mediates the association of meal-related rituals learned in childhood with the avoidance of food waste behavior among Japanese adults.

Study Design, Setting, Participants: Four hundred Japanese adults participated in the web-based cross-sectional questionnaire survey.

Measurable Outcome/Analysis: The following information was included in the questionnaire: participants' background (age, sex, BMI, household size, subjective social status, and dietary change from a year ago), present avoiding food waste behaviors (measured by a sub-scale of validated "sustainable and healthy eating behaviors" questionnaire; 3 items), present appreciation for food (measured by a sub-scale of the Mindful Eating Inventory; 3 items), rituals before and after the meal in childhood (1 item), and advice from parents or educators to refrain from leaving food in childhood (1 item). Mediation analyses with percentile-corrected bootstrap confidence intervals were conducted to calculate the indirect effects adjusted for participants' background.

Results: The participants' mean (SD) age and BMI were 40.0 (11.6) years and 21.9 (3.9) $\mathrm{kg} / \mathrm{m} 2$, respectively (female $=50.0 \%)$. The mean (SD) score of avoiding food waste was 16.0 (4.08; range: 7-21). Appreciation for food mediated the association between meal-related rituals before and after the meal (indirect effects fully adjusted models: $\mathrm{B}=0.085,95 \% \mathrm{CI}=0.009-0.184)$ and advice by parents or educators to refrain from leaving food $(\mathrm{B}=0.101,95 \% \mathrm{CI}=0.016-0.221)$ and avoiding food waste behavior.

Conclusion: Learning experiences from childhood seem to have an influence on present food waste behavior and are mediated by appreciation for food.

Funding: The Foundation for Dietary Scientific Research.

\section{What's the Catch? A Virtual Education Program to Increase Seafood Intake of New Jersey Residents}

Christine Zellers, MPP, zellers@njaes.rutgers.edu, Rutgers Cooperative Extension, DN 703, 4 Moore Rd, Cape May Court House, NJ, 08210-1601; Lauren Errickson, MA, Rutgers Cooperative Extension; Douglas Zemeckis, PhD, Rutgers Cooperative Extension; Joanne Kinsey, MS, CFCS, Rutgers Cooperative Extension; Rachel Tansey, MA, Rutgers Cooperative Extension

Objective: To develop, deliver, and evaluate an extension program designed to increase seafood purchases and consumption.

Use of Theory or Research: According to the Dietary Guidelines for Americans (DGAs), 90\% of consumers fail to meet seafood intake recommendations for maintaining a healthy lifestyle. Reducing barriers such as knowledge and self-efficacy regarding seafood may increase consumption.

Target Audience: New Jersey consumers served by Rutgers Cooperative Extension.

Program Description: The 2-part What's the Catch? (WTC) program communicated responsible, sustainable methods of local fishing and aquaculture (Part 1) and the health and nutritional benefits of seafood (Part 2). Using the DGAs as a framework, WTC-Part 2 shared recommendations to maximize nutritional knowledge and self-efficacy regarding seafood purchase, preparation, and safe handling. Nutrition educators delivered evidence-based information via webinar in October 2020. Nutritional qualities of seafood (low saturated fat and sodium levels, lean protein, and Omega-3 content) and safe levels of mercury intake were featured. Guidance for selecting fresh seafood and minimizing food safety risks, along with preparation methods, recipes, and cooking videos, were provided to increase self-efficacy.

Evaluation Methods: Program participants $(\mathrm{N}=64)$ were asked to complete a quantitative post-attendance Qualtrics survey. Descriptive statistics assessed usual seafood intake frequency, self-reported knowledge improvements, and likelihood of behavior change.

Results: Twenty-one participants (33\%) completed the survey. Most $(66.6 \%)$ consume seafood twice weekly or more (meeting the DGAs), yet $57.1 \%$ were highly likely to consume more seafood and 33\% somewhat likely to increase intake post-program. Nearly $62 \%$ of participants were highly likely, and $28.6 \%$ somewhat likely, to purchase more local seafood. Most participants strongly or somewhat agreed they had improved understanding of the DGAs $(57.1 \%, 38.1 \%)$, mercury $(47.6 \%, 42.9 \%)$, nutrition $(66.7 \%, 23.8 \%)$, and preparation/purchasing $(76.2 \%$, $19.0 \%)$ regarding seafood.

Conclusion: Program outcomes demonstrate that the broad range of seafood topics presented during WTC encouraged consumption by participants. Increased knowledge and self-efficacy through nutrition education likely motivated consumers to partake in healthier behavior.

Funding: NIFA. 